\title{
With Similar Visual Angles, Larger Displays Improve Spatial Performance
}

\author{
Desney S. $\operatorname{Tan}^{\dagger}$, Darren Gergle ${ }^{\dagger}$, Peter G. Scupelli ${ }^{\dagger}$, Randy Pausch ${ }^{\dagger}$ \\ ${ }^{\dagger}$ School of Computer Science, ${ }^{\star}$ School of Design \\ Carnegie Mellon University \\ 5000 Forbes Avenue \\ Pittsburgh, PA 15213, USA \\ \{desney,dgergle\}@cs.cmu.edu,pgs@andrew.cmu.edu,pausch@cmu.edu
}

\begin{abstract}
Large wall-sized displays are becoming prevalent. Although researchers have articulated qualitative benefits of group work on large displays, little work has been done to quantify the benefits for individual users. We ran two studies comparing the performance of users working on a large projected wall display to that of users working on a standard desktop monitor. In these studies, we held the visual angle constant by adjusting the viewing distance to each of the displays. Results from the first study indicate that although there was no significant difference in performance on a reading comprehension task, users performed about $26 \%$ better on a spatial orientation task done on the large display. Results from the second study suggest that the large display affords a greater sense of presence, allowing users to treat the spatial task as an egocentric rather than an exocentric rotation. We discuss future work to extend our findings and formulate design principles for computer interfaces and physical workspaces.
\end{abstract}

Categories and Subject Descriptors: H.5.2 [Information Interfaces and Presentation]: User Interfaces - Screen design, User-centered design, Graphical user interfaces; J.4 [Social and Behavioral Sciences]: Psychology

General Terms: Human Factors, Performance

Keywords: Large display, field of view, visual angle, spatial task, immersion, presence

\section{INTRODUCTION}

We believe that there is an emerging trend in the workplace towards multiple displays, which have the potential to provide abundant display space distributed throughout the environment. Such workplaces typically include several types of displays, each with characteristics that may make it more or less suitable for certain tasks. To explore how each display fits into these new workplaces, we constructed a

Permission to make digital or hard copies of all or part of this work for personal or classroom use is granted without fee provided that copies are not made or distributed for profit or commercial advantage and that copies bear this notice and the full citation on the first page. To copy otherwise, or republish, to post on servers or to redistribute to lists, requires prior specific permission and/or a fee.

CHI 2003, April 5-10, 2003, Ft. Lauderdale, Florida, USA

Copyright 2003 ACM 1-58113-630-7/03/0004 ...\$5.00. system we call the Display Garden. This system is a rapidly configurable collection of physical display devices such as whiteboards and pin-up space, audio displays, mobile LCD panels, and large projection displays on various surfaces in the room. In this paper, we discuss one component of this system, the large projection displays. Researchers have previously realized that "when a display exceeds a certain size, it becomes qualitatively different" [25]. Here, we report results from two studies we conducted to quantify some of the benefits of working on large displays.

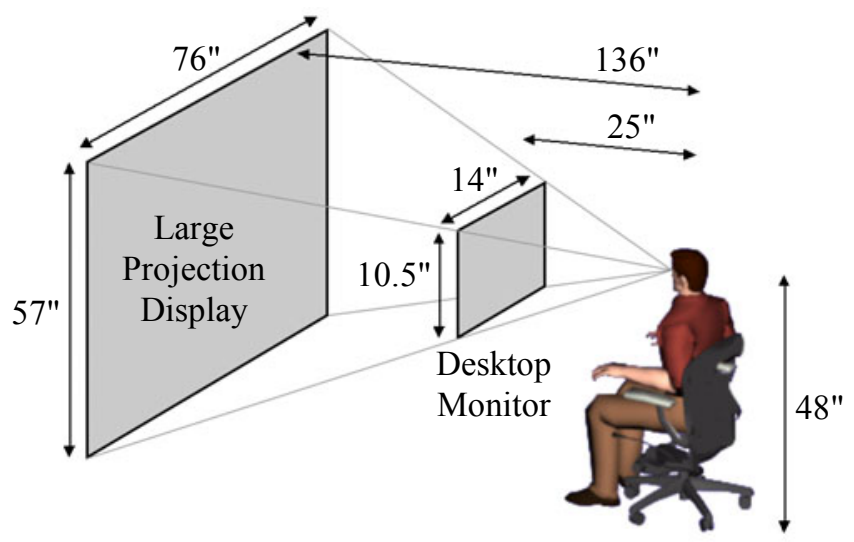

Figure 1. We maintained a constant visual angle for each of the two displays. Only size and distance to the user changed.

Our first study included a reading comprehension task and a spatial task. Users performed both tasks on a large wall projected display and on a smaller desktop monitor. Because we were interested in the effects of size and distance, the visual angle subtended from the user to each of the two displays was kept constant (see Figure 1). Although we found no significant differences in reading speed or comprehension levels, we did find that users performed about $26 \%$ better on a task requiring mental rotation when working on the large display. We hypothesized that this effect was due to a greater sense of presence afforded by the large display. This sense of presence allowed users to use an egocentric strategy, in which they imagined rotating their bodies within the environment, rather than an exocentric one, in which they 
imagined rotating the environment around themselves [4,29]. In order to validate this hypothesis, we ran a second study that presented the same task with two different representations, one which did not bias users to use either an egocentric or exocentric strategy, and the other which biased users toward an exocentric strategy. We found that the benefits extended only to the task that allowed easy access to egocentric representation and strategy.

Our results suggest that, given a constant visual angle, the size and distance of a display may affect perception and performance in spatial orientation tasks. The contribution of these findings is significant because we have identified and begun to quantify new characteristics that, along with traditional ones such as brightness, contrast, position, and field of view, must be considered in designing large-scale information display systems.

\section{RELATED WORK}

In constructing complex workspaces, researchers have pursued the use of large displays for collaborative tasks $[7,12,20,23,27]$. Large displays in these settings are easy for all users to see and interact with [14], providing a conduit for social interaction. Some of these researchers have begun to document performance increases for groups working on large displays [10].

While much work has focused on collaboration, less has been done to design for and objectively measure individual gains on large displays. To this end, several researchers have explored the use of large displays as a means to provide contextual information to the individual. For example, Baudisch et al. [2] provide a large low-resolution overview of the working context around a smaller highresolution focal screen.

Other researchers have realized that large displays may afford users a greater sense of presence, which may benefit performance of certain tasks. Slater \& Usoh [22] define presence as "a state of consciousness, the (psychological) sense of being in the virtual environment." They distinguish it from immersion, which they define to be an objective description of the technology, describing "the extent to which computer displays are capable of delivering ... illusion of reality to the senses of the human participant." In most current models, the sense of presence is seen as the direct outcome of immersion.

The more inclusive, extensive, surrounding, and vivid the display, the higher the potential of presence [3]. In fact, when users are present in Virtual Environments (VEs), the location of their physical bodies are often construed as being contained within that space rather than looking at it from the outside. It is in this state that users are most effective in VEs. Tan et al. [26] utilize large peripheral projection displays to show different scenes of distinct 'places' that the user can use as cues to remember more information. They claim that the greater the sense of presence invoked in the user by the large display, the better the memory for learned information. They do not, however, articulate explanations for the increased sense of presence on the large display. Here we discuss several factors that may cause this effect.

One of these factors is field of view (FOV). Large displays are not often placed at a distance that is proportional to their increase in size over small displays. Due to space constraints, they are typically relatively closer and cast a larger retinal image, thus offering a wider FOV. It is generally agreed that wider FOVs can increase "immersion" in VEs $[16,19]$. Researchers in the entertainment industry have reported that larger displays filling a wider FOV can increase the level of involvement experienced by users [6]. Czerwinski et al. [8] report evidence that a wider field of view offered by a large display leads to an increased sense of presence and improved performance in 3D navigation tasks, especially for females. They document prior literature suggesting that restricting FOV leads to negative impacts on perceptual, visual, and motor performance in various tasks, possibly because users find it difficult to transfer real world experience and cognition into the VE. Arthur [1], in his doctoral dissertation, provides a comprehensive review of the effects of FOV on task performance, especially as carried out in head-mounted displays.

Despite the large amount of work done in comparing FOVs, few researchers have isolated the effects of physical size and distance on task performance or the sense of presence. To examine the psychophysical effects of distance and size, Chapanis \& Scarpa [5] conducted experiments comparing the readability of physical dials at different distances. They used dials of different sizes and markings that were proportional to the viewing distance so as to keep visual angles constant. Perhaps surprisingly, they found that beyond 28 inches away, dials adjusted to subtend the same visual angle were read more easily at greater distances. The effects they found were, however, relatively small.

In a more recent study, Patrick et al. [18] compared various display technologies, with comparable visual angles, and their effects on the spatial information users acquired by navigating through a VE. They found that while users performed significantly worse in forming cognitive maps and remembering the environment on a desktop monitor, they performed no differently using a head-mounted display or a large projection display. They attributed part of this effect to a higher level of presence afforded by the size of the projection display, which compensated for the immersion afforded by the head tracking. In our work, we further explore the effects of display size and distance, with constant FOV or visual angle, on users' sense of presence and performance on various tasks.

One of the task domains we chose was that of mental rotations. Presentation, degree of immersion, and level of performance have been extensively measured for such tasks. In their work, Suzuki \& Nakata [24] had six students 

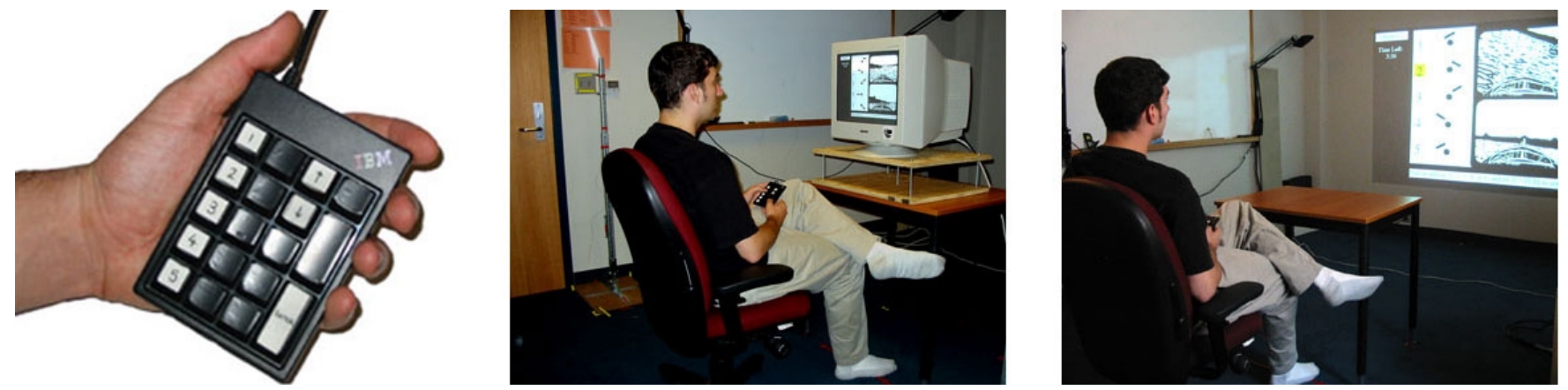

Figure 2. Input device used (left); User working on the small (middle) and large (right) displays.

perform a mental rotation task similar to that of Shepard \& Metzler [21]. Users were asked to judge whether pairs of figures, each of which had been rotated to different degrees, were identical in shape or not. They found, as Shepard \& Metzler did, that mean reaction times increased linearly with the angular difference between figures. They also discovered that visual angle, which corresponds to retinal size, of the objects affected the speed of rotation. However, in this study, viewing distance, given constant visual angle, did not seem to affect reaction times.

Building on this work, Wraga et. al [29] measured spatial knowledge by the time it took users to update their orientation after changing it. Results showed that users were faster at spatial updating when they imagined rotating themselves in the environment rather than when rotating the environment around themselves. Carpenter \& Proffitt [4] extended these findings by examining egocentric rotations in each of the three possible rotation planes. They replicated the finding that egocentric rotation, or rotating one's self, was faster than exocentric rotation, rotating the environment. However, this was true only of planes in which users had experience rotating or locomoting. Tlauka [28] found similar results by comparing rotations of images presented horizontally or vertically.

Another task we chose to evaluate was a reading comprehension task. As with mental rotations, presentation and level of performance for reading comprehension tasks have been well researched and documented [9,17]. Researchers have shown that many factors affect the readability of text, or ease with which meaning of text can be comprehended, on computer screens. Such factors include features of characters, formatting, contrast and color, as well as other dynamic aspects of the screens.

Despite the deep understanding this body of literature offers, there seems to be a gap in work isolating the effects of display size and distance, given a constant visual angle, for performance on tasks. Because of the emergence of large displays in the workplace and in consideration of everyday desktop computing tasks, we decided to evaluate how display size affects performance on spatial orientation and reading comprehension tasks.

\section{EXPERIMENT 1}

\section{Participants}

Twenty-four (12 female) college students, who were intermediate to experienced computer users, participated in the study. We screened users to be fluent in English and to have normal or corrected-to-normal eyesight. The average age of users was 25.4 (25.5 for males, 25.3 for females), ranging from 19 to 32 years of age. Users were paid for their participation.

\section{Materials}

We used two displays, an Eiki Powerhouse One LCD projector and an 18" Sony Trinitron E400 CRT monitor. Both displays ran at a resolution of $1024 \times 768$, and were calibrated to be of roughly equivalent brightness and contrast. We mounted the projector from the ceiling and projected onto a white plaster wall. The image projected on the wall was 76" wide by 57" tall. The image on the monitor was adjusted to be exactly 14 " wide by 10.5 " tall (see Figure 2). We set the two displays up so that when either display was viewed from a specific spot in the room, the visual angle, and hence the size of the retinal image, would be identical (see Figure 1). We assumed a comfortable viewing distance of 25 " for the monitor. In order to get an image of identical perceived size, the projection was set up to be 136" away from the user. The center points of both displays were set to be at eye-height, assumed to be 48 " above the ground. The position of the monitor was carefully marked so that it could be moved in and out as necessary. To mark the spot around which the user's eyes should be centered, we stretched fishing line from two stands, one on either side of the user. A mark in the center of the line indicated the exact spot in the room where the retinal images would be of identical size.

The study was run on a single $800 \mathrm{MHz}$ Dell computer equipped with a dual-headed nVidia GeForce2 MX graphics card. We controlled the activation and deactivation of the displays using the Windows 2000 multiple monitor API so that only one display was active at any given time. The user provided input using an IBM USB numeric keypad with keys we had marked for the experiment (see Figure 2). 


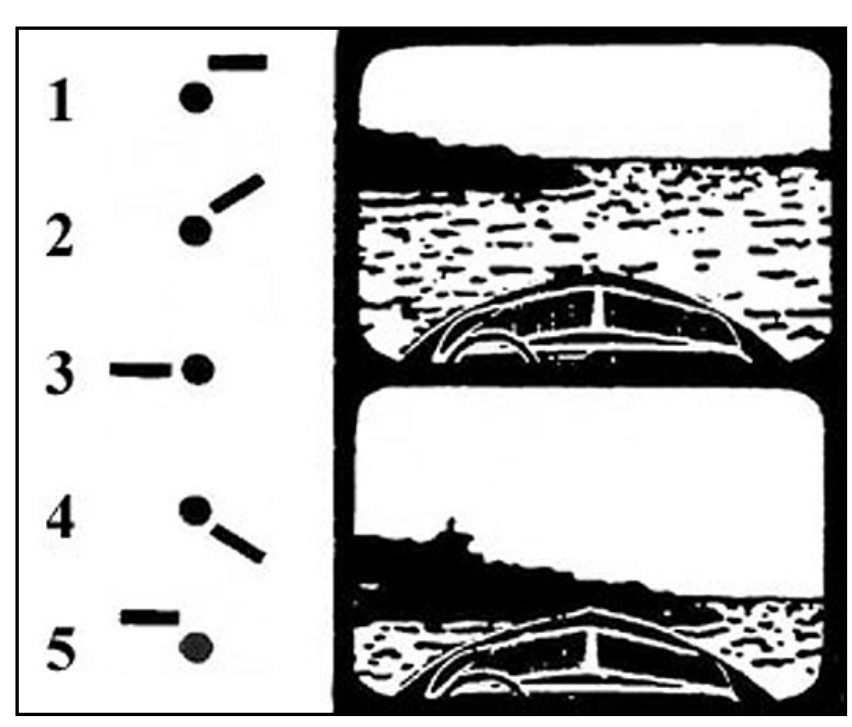

Figure 3. Sample question from the GuilfordZimmerman Spatial Orientation test. The correct answer is number 5 .

\section{Procedure}

After users filled out a background survey, we gave them the input device and had them sit comfortably in the chair. We adjusted the height and position of their chair so that the center of their eyes was as close to the marked fishing line as possible. Once they were viewing the displays from the spot in the room that provided retinal images of identical size, we removed the fishing line. Users were asked not to further adjust the chair or move it around.

\section{Guilford-Zimmerman Spatial Orientation Task}

To evaluate the effects of display size on spatial performance, we utilized the Guilford-Zimmerman Spatial Orientation test [13]. This test has been well validated and researchers have shown that results from this test correlate highly with wayfinding ability [15].

Each question in this test contained two pictures seen from the prow, or front, of a boat along with a multiple choice answer key (see Figure 3). The user was asked to imagine that each picture was taken with a camera fastened rigidly to the boat so that the camera bobbed up and down and slanted with the boat. First, the user looked at the top picture to see where the boat was initially heading. This heading is represented by the dot in the answer key. Next, the user looked at the bottom picture and determined the change in orientation of the boat. The line in each of the possible answers represents the new orientation of the boat relative to the previous heading. Finally, the user selected the answer with the number keys, confirmed the answer with the enter key, and proceeded to the next question.

We gave users the paper-based instructions that were provided with the standard Guilford-Zimmerman test. They then tried 3 practice questions on the large display. For these questions, the system provided users with immediate feedback explaining the correct answers. After they had performed the practice questions, users proceeded to perform the test on the small and the large display, which we will refer to as Display Size. They were not given feedback for these questions. 60 questions were randomized and broken into two sets. Users had 5 minutes to answer 30 questions in each of the two conditions, and were told to perform as quickly and accurately as possible. The order of Display Size was counterbalanced across users. Users had a 30 second rest interval between each condition.

\section{Reading Comprehension Task}

We also tested subjects on a reading comprehension task in the two Display Size conditions. Based on the normalized average scores for the specific passages as well as pilot test data, we chose a suite of 7 passages from practice GRE tests [11] that had relatively similar levels of difficulty. Each of these passages contained about 460 words, or 56 to 60 lines of text as laid out in paper-based GRE format. Each passage came with a set of 7 questions that the user answered after reading the passage.

We gave users verbal instructions on how to scroll through the passage and to answer questions. Then they performed the task with a practice passage on the large display with medium-sized text. We instructed them to work through the questions quickly but accurately. When they had finished the practice passage, they read the rest of the passages and answered questions in each of the 6 conditions, created by presenting text in a given Font Size (Small: 10 point vs. Medium: 14 point vs. Large: 18 point) on each of the Display Size conditions (Small vs. Large). Font Size and Display Size were counterbalanced separately. Users had a 30 second rest interval between passages.

\section{Post-test Preference Questionnaire}

After users completed the tests, they filled out a questionnaire indicating their preference for the conditions in each of the tasks. They were also encouraged to comment on their opinion of the displays.

\section{Results}

We present the results from Experiment 1 in three parts. First we explore performance on the spatial orientation task, then the performance on the reading comprehension task, and finally we investigate preference measures collected at the end of the study.

\section{Spatial Task Performance}

We analyzed data for the spatial orientation task at the summary level. The dependent variable was the percentage of correct responses (number correct / number attempted) ${ }^{1}$. Time differences between different Display Sizes were not significantly different and were therefore dropped from the final models (levels of significance did not change either

\footnotetext{
${ }^{1}$ We use percentage of correct answers as the dependent variable since it is a straightforward and intuitive measure. However, we also examined the sum of correct responses, controlling for time, and found nearly identical results.
} 


\section{Percent Correct by Display Size}

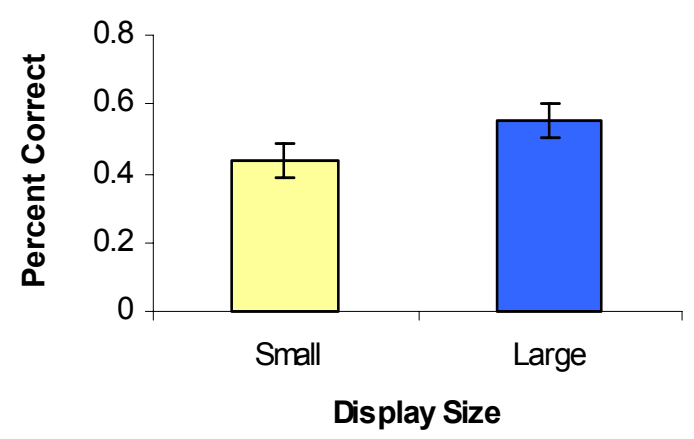

Figure 4. Main effect of Display Size. Large display aided users in the Guilford-Zimmerman test.

way). We analyzed the percentage of correct answers with a 2 (Display Size) x 2 (Position) x 2 (Gender) repeated measures analysis of variance (RM-ANOVA). We analyzed Gender and Position as between-subjects factors and Display Size as a within-subject factor.

We found a significant main effect of Display Size $(\mathrm{F}(1,20)=9.470, \mathrm{p}=.006)$ with the large display resulting in a higher percentage of correct responses on average (Small $=43.8 \%$, Large $=55.4 \%$; see Figure 4). We also observed a significant main effect of Gender $(\mathrm{F}(1,20)=5.072, \mathrm{p}=.035)$, with males producing a higher percentage of correct responses than females on average (Female $=38.7 \%$, Male $=60.4 \%$ ). None of the 2-way or 3-way interaction effects were significant. While Czerwinski et al. [8] suggest that females benefit significantly more than males in 3D navigation tasks using displays with wide fields of view, we saw no such effect for Display Size in our studies. The larger display seems to have benefited both males and females equally.

Since this was a timed task, an alternate explanation for these findings may include a speed-accuracy tradeoff. However, a separate analysis confirmed there was no difference in time spent per question in the two conditions. In addition, we implicitly controlled for the effect of time by taking the percent of answered questions rather than raw scores (see Footnote 1).

Overall, we found a significant improvement in the percent of correct responses on the spatial task for users working on the larger wall display. Keep in mind that while the absolute size of the image was larger, the perceived (or retinal image size) was kept nearly constant regardless of Display Size.

\section{Reading Comprehension Performance}

In the reading comprehension task, we again analyzed data at the summary level. We used the number of correct responses for each condition as the dependent variable. We performed an RM-ANOVA in which Position, Display Size, and Font Size were repeated and Time to complete the question was a covariate. We included all 2-way and 3-way interactions in the analysis. Because each user participated in multiple trials, within observations were not independent. User was modeled as a random effect.

Overall, performance in the reading comprehension task did not differ across the conditions. We found no difference between small $(\mathrm{M}=3.86)$ and large $(\mathrm{M}=4.01)$ Display Sizes $(\mathrm{F}(1,106)=.367, \mathrm{p}=.546)$. Similarly, we found no difference between the small $(\mathrm{M}=3.84)$, medium $(\mathrm{M}=3.96)$ and large $(\mathrm{M}=4.01) \quad$ Font $\quad$ Sizes $\quad(\mathrm{F}(2,106)=.176, \mathrm{p}=.839)$. The interaction between Display Size and Font Size was not significant, $(\mathrm{F}(2,106)=1.159, \mathrm{p}=.3178)$.

We were unable to reject the null hypothesis that the displays were equal for performance on the reading comprehension task. Thus, while we did find differences on the spatial orientation task, we found no evidence to suggest that the performance on reading comprehension was different on either of the two Display Sizes, regardless of Font Size.

\section{Preference Data}

In addition to the performance data, we gathered preference data from users at the conclusion of the study. The questions were asked on a 5-point Likert scale of $1=$ "Strongly prefer small display" to $5=$ "Strongly prefer large display".

Given the performance difference we found on the spatial task, we were primarily interested in user preference for this task. Users significantly preferred the large display for both 'Ease of Seeing,' $(\mathrm{M}=3.61, \quad \mathrm{p}=.019)$ and 'Overall Preference,' $(\mathrm{M}=3.50, \mathrm{p}=.045)$. They marginally preferred the large display for their 'Confidence in the Rotation Task,' $(\mathrm{M}=3.43, \mathrm{p}=.066)$. Users showed no significant preference for display in the reading comprehension task.

\section{Summary}

While we found a difference in performance on the spatial orientation task presented in this study, we did not know exactly what had caused the performance benefits. Was it because the larger display provided a greater absolute difference in scale, provided a more immersive environment that encouraged egocentric representation and strategy, or did it have something else to do with the surroundings? In order to further explore the reason behind such a dramatic improvement in performance (approximately a $26 \%$ increase), we decided to run a second study to investigate the difference.

\section{EXPERIMENT 2}

\section{Participants}

Twenty-four (14 female) college students, who did not participate in the first study, participated in this study. As before, we screened users to have normal or corrected-tonormal eyesight. The average age of users was 24.1 (25.4 for males, 23.2 for females), ranging from 18 to 56 years of age. Users were paid for their participation. 


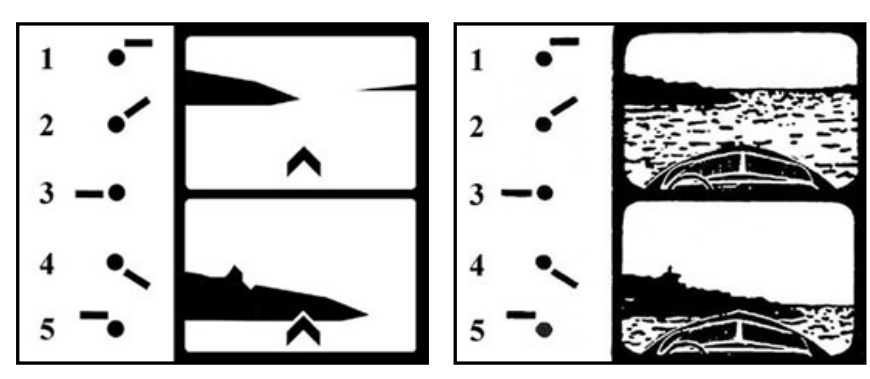

Figure 5. New Shape test with abstract representation (left); Original Guilford-Zimmerman test with boat theme (right).

\section{Materials and Procedure}

We used the same setup as in Experiment 1. For this study, we created a derivative of the Guilford-Zimmerman test, which we call the Shape test. The original GuilfordZimmerman test was carefully crafted not to bias a user into any particular strategy. We designed our new Shape task to bias the user into an exocentric strategy. Although we could have used a test that already does this, such as the ShepardMetzler test, we decided on creating the Shape test to keep baseline performance levels between the two groups similar. To create this test, we did two things. First, we mimicked the scenes presented in the original test with abstract shapes (see Figure 5). Second we edited the instructions so that rather than asking users to imagine themselves looking through a camera mounted on a boat that was bobbing and rocking, we had them imagine themselves looking through a camera that was rigidly mounted on the ground in front of a painting that was moving and turning. After balancing for gender, each participant was randomly assigned to one of the two Task Types: original Guilford-Zimmerman test or our new Shape test. The procedure for each test was the same as in Experiment 1, minus the reading test.

\section{Results}

We present results from Experiment 2 in two parts. First we explore performance on the spatial orientation task; then we examine the preference data.

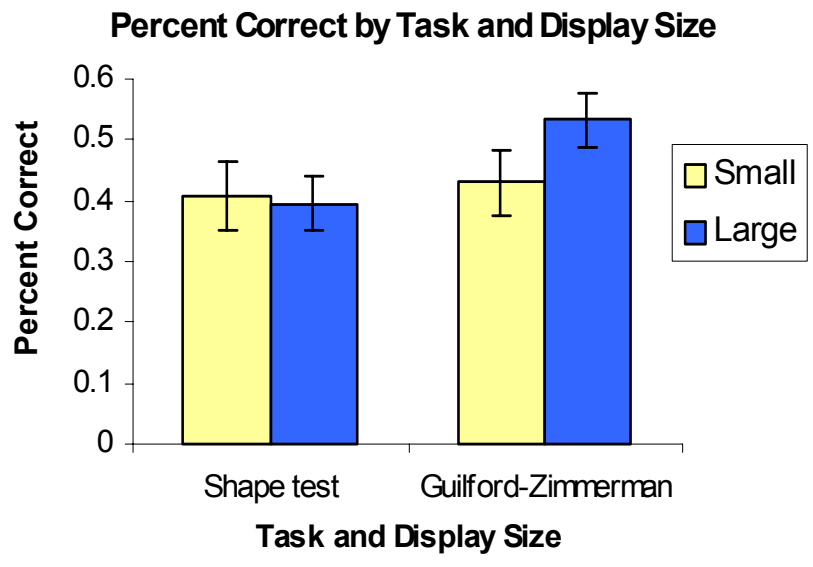

Figure 6. The large display was better for the Guilford-Zimmerman test but not the Shape test.

\section{Egocentric and Exocentric Spatial Task Performance}

We modeled the data as we did for the spatial orientation task in the previous study. Again we examined data at the summary level and used the percentage of correct responses (number correct / number attempted) as the dependant variable. We analyzed the percentage of correct responses with a 2 (Display Size) x 2 (Task Type) x 2 (Position) RMANOVA. We analyzed Task Type and Position as betweensubjects factors and Display Size as a within-subject factor.

Overall, we found a marginally significant effect of Display Size $(\mathrm{F}(1,21)=3.296, \mathrm{p}=.084)$ with the large display resulting in a marginally higher percentage of correct responses, on average (Small $=41.8 \%$, Large $=46.3 \%$ ). We did not necessarily expect this main effect to be as strong as in Experiment 1, particularly if the Shape test showed no improvement in the large display condition. A more detailed investigation revealed that this was indeed the case. We found a significant interaction between the Display Size and Task Type $(\mathrm{F}(1,21)=5.512, \mathrm{p}=.0288)$ demonstrating that the benefits of the large display were significantly greater for the Guilford-Zimmerman task (Small=42.9\%, Large $=53.2 \%$ ) than for the Shape task (Small $=40.8 \%$, Large $=39.5 \%$ ). Thus, the large display provided for better performance in the Guilford-Zimmerman task, but it did not appear to help users with the Shape task (see Figure 6).

These results provide partial replication of the findings from Experiment 1, with additional insight into the hypothesis that large displays facilitate egocentric rotation.

\section{Preference Data}

As in Experiment 1, we gathered preference data from the participants at the conclusion of this study. The same questions were asked using the same 5-point Likert scale.

The merged preference data for both the GuilfordZimmerman and Shape groups were not significantly in favor of the large display. We explored whether or not the different task groups viewed the value of the displays differently (see Figure 7). We found that users performing the Guilford-Zimmerman task preferred the large display for 'Confidence in the Rotation Task,' $(\mathrm{t}(22)=-2.250$, $\mathrm{p}=.0348$ ) and marginally preferred the large display for 'Overall Performance,' $(\mathrm{t}(22)=-1.773, \quad \mathrm{p}=.090)$, in comparison to the users doing the Shape task. However, they did not rate the large display as any better than Shape test users did for 'Ease of Seeing,' $(\mathrm{t}(22)=-1.089, \mathrm{p}=.288)$.

\section{GENERAL DISCUSSION}

Overall, our results demonstrated a benefit to working on a large display. We reported two experiments examining performance on various tasks done on a large versus a small display, both observed with a constant visual angle. The first experiment demonstrated the benefits of the larger display for performing a spatial orientation task, but found no evidence to suggest that reading comprehension was better in either display condition. 


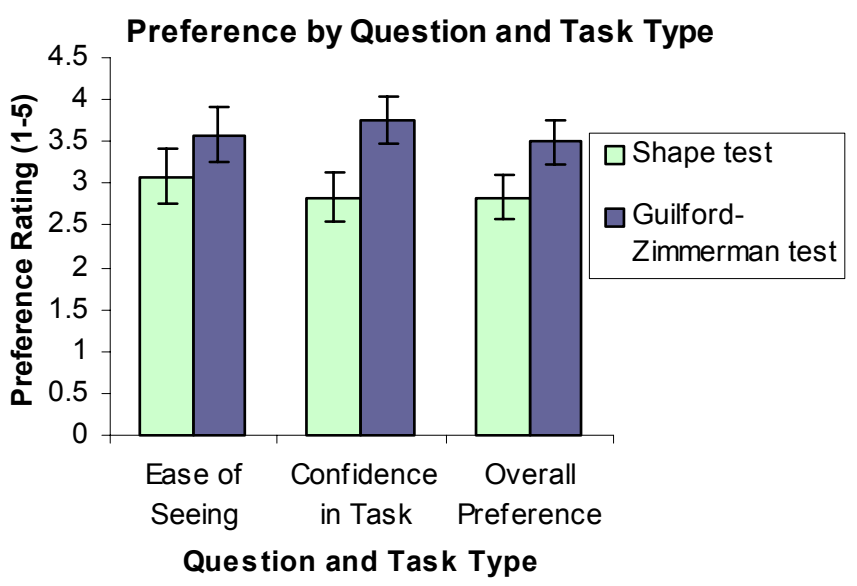

Figure 7. Users in the Guilford-Zimmerman test significantly preferred the large display $(>3)$ for 'Confidence in Task' and 'Overall Preference', but not for 'Ease of Seeing.'

The fact that we found differences in the spatial task but not the reading comprehension task led us to believe that there may be an interaction between the task and the display size. We hypothesized that the performance difference on the spatial orientation task was due to the way the image was perceived and thus the strategy with which users performed the task. Note that users could perform the task egocentrically, imagining rotating their bodies in the environment, or exocentrically, imagining the environment rotating around them.

The instructions for the Guilford-Zimmerman test are carefully worded so as not to bias strategy choice one way or another. This allows users to either imagine themselves on the boat looking through the camera as the boat moves within the environment (egocentric), or outside the environment as the boat rotates within it (exocentric). We believed that as users became more immersed in the task on the large display, they were more likely to adopt the egocentric strategy. Since egocentric rotations have been shown to be quicker [4,29], this would explain the performance increase we observed on the large display.

To test our hypothesis, we created a similar test, the Shape test, by replacing all environmental cues in the GuilfordZimmerman test with abstract shapes and by changing the instructions to bias the user into performing the task exocentrically. Note that we could just as easily have used a test that already does this, such as the Shepard-Metzler test, but decided on the Shape test to keep baseline performance levels between the two groups similar. If our hypothesis was correct, and users adopted an exocentric strategy, then users performing this task on the large display would perform no better than on the small display. This would be similar to the results found by Suzuki and Nakata [24], in which the larger display had no effect on performance. Results show that this was indeed the case. Users did not perform significantly different on the two displays with the
Shape task, but again showed a performance increase for the original Guilford-Zimmerman task.

User satisfaction ratings strongly support performance results in that users performing the Guilford-Zimmerman test significantly preferred the large display to the small. Users performing this task in the second study also significantly preferred the large display for 'Confidence in Rotation' and 'Overall Performance,' as compared to users doing the Shape test. However, ratings for 'Ease of Seeing' did not differ for the two tasks, suggesting that the effects may not have been driven solely by characteristics of the display, such as brightness or contrast.

\section{CONCLUSION AND FUTURE WORK}

We ran two studies to compare the performance of users working on a large projected wall display to that of users working on a standard desktop monitor. In these studies, we held the visual angle constant by adjusting the viewing distance to each of the displays. Results from the first study show that although there was no significant difference in performance on a reading comprehension task, users performed better on the Guilford-Zimmerman spatial orientation task done on the large display. Results from the second study suggest that the large display affords a greater sense of presence, leading users to perform the task using a more efficient egocentric strategy.

To extend this work, we would like to explore in more detail other spatial tasks that may benefit from larger displays. For example, 3D navigation and wayfinding might reap large benefits from having a user who feels more present in the environment and is thus able to perform spatial orientation tasks more quickly or easily. We would also like to build a theoretical framework of such tasks so that we may further inform the design both of spatial tasks to be presented on large displays, but also of display systems that may be built to run these tasks. Implications of these findings may prove critical to educational and work settings in spatial disciplines, such as architecture and engineering fields that use computer-aided design.

\section{ACKNOWLEDGEMENTS}

We thank Dennis Proffitt and his Perceptual Psychology Lab, the Stage 3 Research Lab, and Azizan Aziz for stimulating discussions on multiple display systems. Susan Fussell and Robert Kraut provided insight into the analyses. Sarah Hatton coordinated the artwork in this paper.

\section{REFERENCES}

1. Arthur, K.W. (2000). Effects of field of view on performance with head-mounted displays. Doctoral Dissertation, University of North Carolina, Chapel Hill, NC.

2. Baudisch, P., Good, N., Belloti, V., \& Schraedley, P. (2002). Keeping things in context: A comparitive evaluation of focus plus context screens, overviews, and zooming. Proceedings of CHI 2002, 259-266. 
3. Bystrom, K.E., Barfield, W., \& Hendrix, C. (1999). A conceptual model of the sense of presence in virtual environments. Presence: Teleoperators and Virtual Environments, 8(2), 241-244.

4. Carpenter, M., Proffitt, D. (2001). Comparing viewer and array mental rotations in different planes. Memory \& Cognition, 29, 441-448.

5. Chapanis, A., Scarpa, L.C. (1967). Readability of dials at difference distances with constant viewing angle. Human Factors, 9(5), 419-426.

6. Childs, I. (1988). HDTV-putting you in the picture. IEE Review, 34(7), 261-265.

7. Chou, P., Gruteser, M., Lai, J., Levas, A., McFaddin, S., Pinhanez, C., Viveros, M., Wong, D., \& Yoshihama, S. (2001). BlueSpace: Creating a personalized and context-aware workspace. IBM technical report, $\mathrm{RC} 22281$.

8. Czerwinski, M., Tan, D.S., Robertson, G.G. (2002). Women take a wider view. Proceedings of CHI 2002, 195-202.

9. Dillion, A. (1992). Reading from paper versus screens: A critical review of the empirical literature. Ergonomics, 35(10), 1297-1326.

10. Dudfield, H.J., Macklin, C., Fearnley, R., Simpson, A., \& Hall, P. (2001). Big is better? Human factors issues of large screen displays with military command teams. Proceedings of People in Control 2001, 304-309.

11. Educational Testing Service. (1994). Practicing to take the GRE test. New Jersey: ETS.

12. Elrod, S., Bruce, R., Gold, R., Goldberg, D., Halasz, F., Janssen, W., Lee, D., McCall, K., Pederson, E., Pier, K., Tang, J., \& Welch, B. (1992). Liveboard: A large interactive display supporting group meetings, presentations and remote collaboration, Proceedings of CHI 1992, 599-607.

13. Guilford, J.P., Zimmerman, W.S. (1948). The Guilford-Zimmerman Aptitude Survey. Journal of Applied Psychology, 32, 24-34.

14. Guimbretière, F. (2002). Fluid interaction for high resolution wall-size displays. Doctoral Dissertation, Stanford University, Stanford, CA.

15. Infield, S.E. (1991). An investigation into the relationship between navigation skill and spatial abilities. Doctoral Dissertation, University of Washington, Seattle, WA. Dissertation Abstracts International, 52(5-B), 2800.

16. Lin, J.J., Duh, H.B.L., Parker, D.E., Abi-Rached, H., Furness, T.A. (2002). Effects of field of view on presence, enjoyment, memory, and simulator sickness in a virtual environment. Proceedings of IEEE Virtual Reality Conference 2002, 164-171.
17. Mills, C.B., Weldon, L.J. (1987). Reading text from computer screens. ACM Computer Surveys, 19(4), 329357.

18. Patrick, E., Cosgrove, D., Slavkovic, A., Rode, J.A., Verratti, T., \& Chiselko, G. (2000). Using a large projection screen as an alternative to head-mounted displays for virtual environments. Proceedings of $\mathrm{CHI}$ 2000, 478-485.

19. Prothero, J.D., Hoffman, H.D. (1995). Widening the field of view increases the sense of presence within immersive virtual environments. Human Interface Technology Laboratory Technical Report, University of Washington, Seattle, WA, R-95-4.

20. Raskar, R., Welch, G., Cutts, M., Lake, A., Stesin, L., \& Fuchs, H. (1998). The office of the future: A unified approach to image-based modeling and spatially immersive displays. Proceedings of SIGGRAPH 1998, 179-188.

21. Shepard, R.N., Metzler, J. (1971). Mental rotations of three-dimensional objects. Science, 171(3972), 701703.

22. Slater, M., Usoh, M. (1993). Presence in Immersive Virtual Environments. Proceedings of the IEEE Conference - Virtual Reality Annual International Symposium, 90-96.

23. Streitz, N.A., Geißler, J., Holmer, T., Konomi, S., Müller-Tomfelde, C., Reischl, W., Rexroth, P., Seitz, P., \& Steinmetz, R. (1999). i-LAND: An interactive landscape for creativity and innovation. Proceedings of CHI 1999, 120-127.

24. Suzuki, K., Nakata, Y. (1988). Does the size of figures affect the rate of mental rotation? Perception \& Psychophysics, 44(1), 76-80.

25. Swaminathan, N., Sato, S. (1997). Interaction design for large displays. Interactions, 4(1), 15-24.

26. Tan, D.S., Stefanucci, J.K., Proffitt, D.R., Pausch, R. (2001). The Infocockpit: Providing Location and Place to Aid Human Memory. Workshop on Perceptive User Interfaces 2001.

27. Tani, M., Masato, H., Kimiya, Y., Koichiro, T., \& Futakawa, M. (1994). Courtyard: Integrating shared overview on a large screen and per-user detail on individual screens. Proceedings of CHI 1994, 44-50.

28. Tlauka, M. (2002). Switching imagined viewpoints: The effects of viewing angle and layout size. British Journal of Psychology, 93, 193-201.

29. Wraga, M., Creem, S.H., \& Proffitt, D.R. (2000). Updating displays after imagined object and viewer rotations. Journal of Experimental Psychology: Learning, Memory, and Cognition. 26(1), 151-168. 